\title{
Protocolos de actuación con la exodoncia en pacientes geriátricos antiagregados y anticoagulados
}

\section{Clinical protocols for tooth extraction in geriatric patients treated with anticoagulants and antiplatelet drugs}

\author{
Rubio-Alonso LJ*, Martínez-Rodríguez N**, Cáceres-Madroño E***, \\ Fernández-Cáliz F****, Martínez-González JM******
}

\section{RESUMEN}

El paciente geriátrico presenta un mayor riesgo cardiovascular, siendo la cardiopatía isquémica la causa más frecuente de muerte en los ancianos. Con la edad se producen ciertos cambios en la homeostasis, con un aumento de la coagulabilidad, disminución de la fibrinólisis y un aumento de la agregabilidad plaquetaria. Estos factores unidos a las alteraciones endoteliales y un mayor estasis sanguíneo, conllevan un aumento del riesgo trombótico, y la posibilidad de padecer con mayor frecuencia enfermedades cardiovasculares. El empleo de antiagregantes plaquetarios y anticoagulantes orales ha demostrado ser una terapéutica efectiva tanto en la prevención primaria como secundaria de estos accidentes trombóticos. Los procedimientos quirúrgicos, como son las extracción dentales, en pacientes con tratamiento antitrombóticos, pueden conllevar un aumento del riesgo de sangrado tanto durante su realización como en el periodo postoperatorio. Por otro lado, la supresión de la terapia antitrombótica para reducir este sangrado, puede aumentar el riesgo de desarrollar un accidente tromboembólico, con unas consecuencias fatales para la salud del paciente. La finalidad de este trabajo es revisar los principales fármacos antitrombóticos usados en el momento actual, y dar a conocer las pautas actuales a seguir en el manejo de estos pacientes cuando van a ser sometidos a una extracción dental. Palabras clave: Antiagregantes plaquetarios, anticoagulantes orales, riesgo trombótico, riesgo hermorrágico.

\section{SUMMARY}

Geriatric patient have an increased cardiovascular risk, and ischemic heart disease is the most common cause of death in the ederly. Age results in changes in the homeostasis as increased coagulability, decreased fibrinolysis and increased platelet aggregability. These factors together with endothelial dysfunction and increased blood stasis, involve an increased thrombotic risk and the possibility of having a cardiovascular event. The use of oral anticoagulants and antiplatelet drugs has amply demonstrated its effectiveness in both primary and secundary prevention of thrombotic events. Surgical procedures, such as dental extraction, in patiens with antithrombotic therapy may entail to an increased risk of bledding during the procedure and in the postoperative period. On

* Profesor contratado del Máster de Cirugía Bucal. Hospital Virgen de la Paloma. Madrid. Colaborador Honorífico de Cirugía Bucal. Facultad de Odontología. UCM.

** Profesora contratada del Máster de Cirugía Bucal. Hospital Virgen de la Paloma.Madrid. Profesora de Clínica Integrada de Adultos y Patología Quirúrgica Bucal. Facultad de Ciencias de la Salud. URJC.

*** Profesora contratada del Máster Implantología. Universidad Alfonso X el Sabio. Colaboradora Honorífica de Cirugía Bucal. Facultad de Odontología. UCM.

**** Profesor contratado del Máster Cirugía Bucal. Hospital Virgen de la Paloma. Madrid. Profesor Asociado de Cirugía Bucal. Facultad de Odontología. UCM.

***** Profesor Titular de Cirugía Maxilofacial. Facultad de Odontología. UCM. 
the other hand, the removal of these drugs in order to reduce bledeing may increase the risk of developing a thromboembolic event with fatal consequences for the patient'shealth. The purpose of this paper is to review antithrombotic drugs currently used and protocols for patients on anticoagulation/antiplatelet therapy who need a dental extraction.

Key words: Antiplatelet drugs, anticoagulants drugs, thrombotic risk, hemorrhagic risk.

Fecha de recepción: 2 de mayo 2015.

Aceptado para publicación: 6 de mayo 2015.

Rubio-Alonso LJ, Martínez-Rodríguez N, Cáceres-Madroño E, Fernández-Cáliz F, Martínez-González JM. Protocolos de actuación con la exodoncia en pacientes geriátricos antiagregados y anticoagulados. Av. Odontoestomatol 2015; 31 (3): 203-214.

\section{INTRODUCCIÓN}

La aterosclerosis y sus complicaciones isquémicas cardiovasculares son las causas más comunes de muerte y discapacidad en todo el mundo. La trombosis desempeña un papel primordial en la aparición de eventos isquémicos coronarios y accidentes cerebrovasculares (ACV) (1).

Los fármacos antitrombóticos van a jugar un papel importante en la prevención y tratamiento de la enfermedad cardiovascular.

Un antiagregante plaquetario es un fármaco cuyo principal efecto es inhibir la agregación de las plaquetas y por lo tanto la formación de trombos o coágulos en el interior de las arterias y venas.

La lesión endotelial o la ruptura de una placa aterosclerótica desencadena la adhesión de las plaquetas a la superficie subendotelial, gracias al receptor plaquetario Ia/Ila y las fibras de colágeno, proceso estabilizado por el factor de von Willebrand (FvW). Este primer contacto plaqueta-vaso, origina la activación de la plaqueta, produciendo y liberando sustancias vasoactivas y promotoras de su agregación, tales como el tromboxano A2 (TXA2), generado desde el ácido araquidónico gracias a la acción de la ciclooxigenasa 1 (COX-1), el FvW, el fosfato de adenosina (ADP) y la trombina. La plaquetas activadas sufren cambios en la conformación de su membrana, que activa otros receptores y la coagulación plasmática. El más importante de estos receptores es la glucoproteína Ilb/IIla, a la que se une el fibrinógeno, dando lugar a la formación de puentes entre las plaque- tas activadas que inducen la formación de agregados plaquetarios (Figura 1).

Los anticoagulantes orales son sustancias que inhiben la coagulación de la sangre, creando un estado antitrombótico o prohemorrágico. Actúan inhibiendo la síntesis de factores de coagulación a nivel hepático o directamente inhibiendo algunos factores de coagulación. El número de pacientes anticoagulados en España es de 13,2 por cada 1000 habitantes (2).

La realización de una extracción dental en un paciente sometido a terapia antitrombótica puede conllevar un aumento de sangrado durante y posteriormente a la intervención. La supresión de la terapia antiagregante o anticoagulante para minimizar este riesgo, puede a su vez incrementar el riesgo tromboembólico en forma de accidente cerebrovascular o cardíaco si la medicación es alterada o suspendida.

La finalidad de este trabajo es por un lado dar a conocer los nuevos fármacos antiplaquetarios y anticoagulantes introducidos en la práctica médica, y por otro lado, poder establecer unas pautas de actuación en aquellos pacientes en tratamiento con fármacos anticoagulantes orales y antiagregantes plaquetarios que van a ser sometidos a una extracción dental.

\section{CLASIFICACIÓN Y MECANISMO DE ACCIÓN DE LOS ANTIAGREGANTES PLAQUETARIOS}

Los antiagregantes plaquetarios, en función de su mecanismo de acción, se pueden dividir en cinco grupos (Tabla 1). 


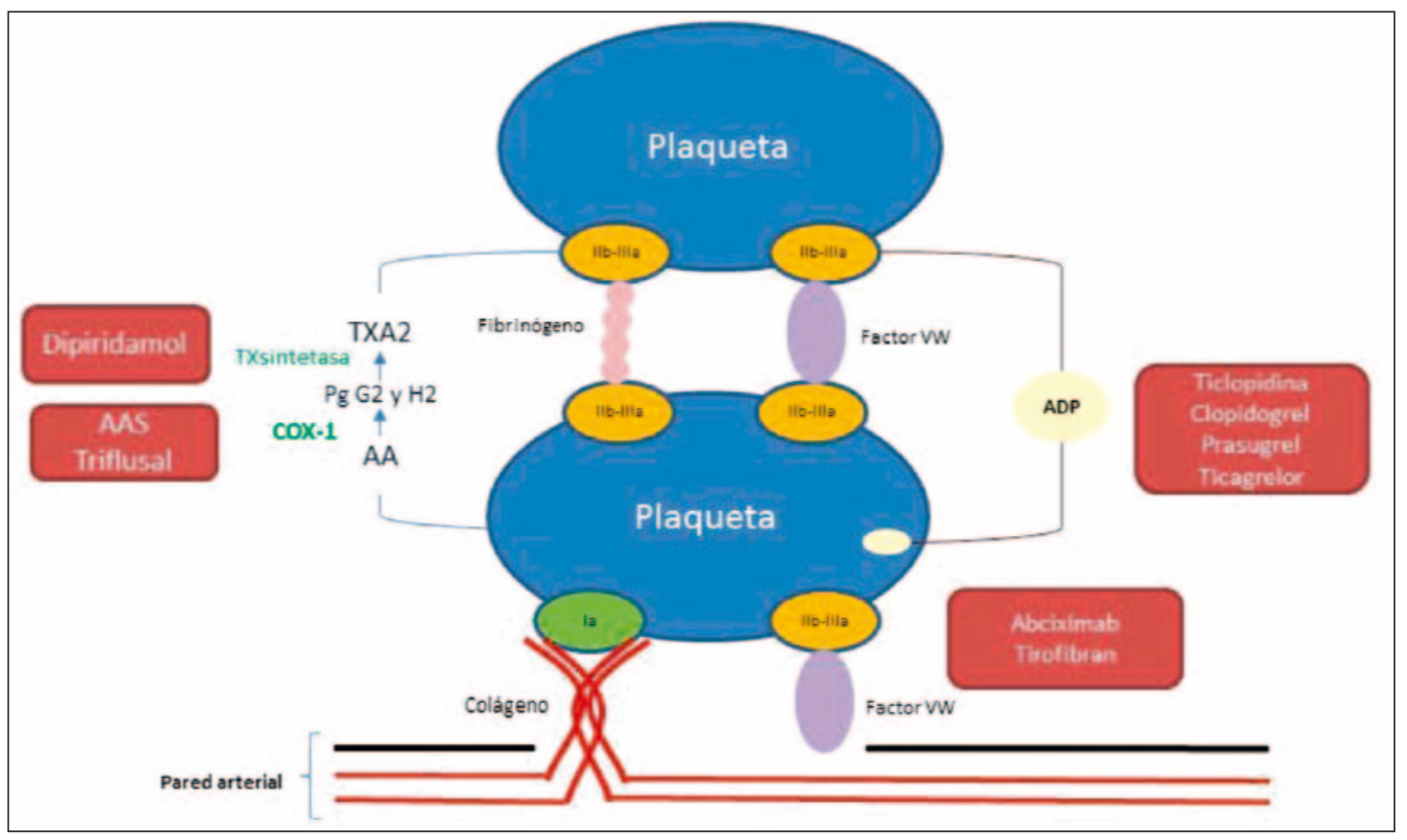

Fig. 1. Esquema del mecanismo de acción de los diferentes agentes antiplaquetarios. Tomado de la Sociedad Valenciana de Medicina familiar y Comunitaria. Antiagregación en atención primaria.

\section{Inhibidores de la ciclooxigenasa}

El ácido acetilsalicílico (AAS) fue el primer fármaco antiplaquetario empleado en clínica (3). Su bajo coste y efectividad demostrada hacen que sea el antiagregante plaquetario más utilizado en la práctica clínica. Su principal mecanismo de acción es la inhibición irreversible de la ciclooxigenasa 1 (COX-1), esta enzima transforma el ácido araquidónico en tromboxano A2, cuya función es la estimulación de la secreción y agregación plaquetaria (4). Su efecto dura la vida de la plaqueta, aproximadamente de 7 a 10 días (5). La dosis habitual es de $75-325$ mg/ día.

trifusal inhibe de forma irreversible la COX-1, aunque tiene también acciones antiplaquetarias por inhibición de la fosfodiesterasa. Tiene una eficacia clínica similar al AAS, pero hay mucha menor experiencia y avales de su efectividad. La dosis habitual es de 300-600 mg/día.

\section{Inhibidores de la fosfodiesterasa}

El dipiridamol produce una inhibición de la fosfodiesterasa, la cual transforma en adenosín monofos- fato cíclico (cAMP) en adenosín monofosfato (AMP), niveles altos de cAMP producen un bloqueo de la actividad plaquetaria, además reduce la adhesión plaquetaria a las prótesis valvulares. Presenta un efecto sobre la adhesión plaquetaria mucho más acentuado que sobre la agregación. No hay datos que demuestren su eficacia clínica aislada, y no existe ninguna evidencia de que sea superior al AAS en ninguna indicación, por lo que es un antiplaquetario en desuso, y sus indicaciones se limitan a su posible utilización en sustitución del AAS en situaciones donde éste no pueda ser utilizado (6). Sus principales efectos adversos son cefaleas, náuseas, epigastralgia y diarrea. Su dosis habitual es $100 \mathrm{mg} /$ 8 horas.

\section{Inhibidores de los receptores de adenosín fosfato (ADP) (receptor P2Y ${ }_{12}$ )}

La ticlopidina constituyó el primer fármaco del grupo de las tienopiridinas utilizado como antiagregante plaquetario, en la actualidad no se utiliza. Se trata de un profármaco que tiene que ser transformado a nivel hepático mediante oxidación en un metabolito activo (tiolactona). Inhibe la agregación plaquetaria inducida por la ADP en forma irreversible a través del 


\begin{tabular}{|c|c|c|c|}
\hline Mecanismo de acción & Nombre del fármaco & Nombre comercial & Vía administración \\
\hline \multirow[t]{4}{*}{$\begin{array}{l}\text { Inhibidor ciclooxigenasa } \\
1 \text { (COX-1) }\end{array}$} & $A A S$ & $\begin{array}{l}\text { Adiro }{ }^{\circledR} \text {, Bioplak }{ }^{\circledR} \\
\text { Tromalyt } ®\end{array}$ & Oral \\
\hline & Trifusal & Disgren ${ }^{\circledR}$ & Oral \\
\hline & Sulfinpirazona & & Oral \\
\hline & Indobufeno & & Oral \\
\hline \multirow{6}{*}{$\begin{array}{l}\text { Inhibidores de } \\
\text { la fosfodiesterasa }\end{array}$} & Dipiridamol & Persantin ${ }^{\circledR}$ & Oral \\
\hline & Ditazol & Ageroplas ${ }^{\circledR}$ & Oral \\
\hline & Trapidil & & Oral \\
\hline & Cilostazol & & Oral \\
\hline & Epoprostenol & & Intravenosa \\
\hline & Ilopost & & Intravenosa \\
\hline \multirow{6}{*}{$\begin{array}{l}\text { Antagonistas del receptor } \\
\text { P2Y }_{12}\end{array}$} & Ticlopidina & Ticlodone ${ }^{\circledR}$, Tiklid ${ }^{\circledR}$ & Oral \\
\hline & Clopidogrel & Plavix ${ }^{\circledR}$, Iscover ${ }^{\circledR}$ & Oral \\
\hline & Prasugrel & Efient ${ }^{\circledR}$ & Oral \\
\hline & Ticagrelor & Brilique ${ }^{\circledR}$ & Oral \\
\hline & Cangrelor & & Intravenosa \\
\hline & Elinogrel & & Oral/intravenosa \\
\hline $\begin{array}{l}\text { Antagonistas glicoproteína } \\
\text { Ilb/IIla }\end{array}$ & $\begin{array}{l}\text { Eptifibatide } \\
\text { Tirofiban } \\
\text { Abciximad }\end{array}$ & $\begin{array}{l}\text { Integrelin }{ }^{\circledR} \\
\text { Aggrastat }{ }^{\circledR} \\
\text { ReoPro }{ }^{\circledR}\end{array}$ & $\begin{array}{l}\text { Intravenosa } \\
\text { Intravenosa } \\
\text { Intravenosa }\end{array}$ \\
\hline \multirow{2}{*}{$\begin{array}{l}\text { Inhibidores del receptor } \\
\text { de la trombina (PAR-1) }\end{array}$} & Vorapaxar & Fase de desarrollo & Oral \\
\hline & Atopaxar & & Oral \\
\hline
\end{tabular}

bloqueo de la unión a receptores específicos (receptor P2Y12). Alcanza su máximo efecto a los 5 u 8 días. La actividad antiagregante depende de su concentración plasmática y dura de 4 a 10 días. Presenta un efecto acumulativo y no tiene un efecto antiagregante inmediato. La ticlopidina tiene efectos colaterales como trombocitopenia, anemia aplásica, púrpura trombocitopénica trombótica, neutropenia e hipercolesterolemia; además, su coste es elevado. Durante las primeras 2 a 3 semanas pueden presentarse, además, alteraciones gastrointestinales como diarrea, náuseas, dispepsia y anorexia, como también erupciones cutáneas.

El clopidogrel es una tienopiridina de segunda generación sin toxicidad que reemplazó gradualmente 
a la ticlopidina. Se trata de un profármaco con una acción antiplaquetaria más marcada que el AAS(7) que inhibe de forma irreversible el receptor P2Y $\mathrm{Y}_{12}$. El efecto máximo de este fármaco se alcanza a las 2-3 horas de su administración. El uso del clopidogrel no es adecuado para el tratamiento inmediato; su efecto antiagregante se mantiene por 7 a 10 días luego de la suspensión del fármaco. Presenta menos efectos secundarios que la ticlopidina, por lo que se prefiere su uso frente a ésta. A día de hoy, el clopidogrel es la tienopiridina de elección, tanto en asociación con AAS como en aquellos pacientes en los que el AAS está contraindicado.

El prasugrel es otro profármaco de una tercera generación de tienopiridinas, con mayor potencia antiagregante, y con una transformación más rápida a metabolito activo, que se une específicamente y de forma irreversible al receptor $\mathrm{P}^{2} \mathrm{Y}_{12}$ del ADP, por lo que su actividad antiplaquetaria dura también de 5 a 10 días. Presenta una activación hepática más rápida y potente que el clopidogrel, con una concentración máxima a los 30 minutos de administración. El prasugrel ha demostrado una mayor eficacia que el clopidogrel en pacientes con síndrome coronario agudo (SCA) (7).

El ticagrelor deriva las ciclopentil-triazolopirimidinas, y a diferencia de las tienopiridinas, inhibe de forma reversible y no competitiva el receptor $\mathrm{P}_{2} \mathrm{Y}_{12}$ del ADP. No es un profármaco, por lo que no requiere un metabolismo para su actividad, por ello presenta una acción antiplaquetaria más potente y rápida $(1,5-2$ horas tras su ingestión) que el clopidogrel (8), y su efecto antiagregante perdura de 5 a 7 días (9).

Cangrelor es un análogo del ATP; se administra por vía endovenosa y es un inhibidor directo, reversible y competitivo del receptor P2Y12. Tiene un inicio rápido y una vida media corta, de aproximadamente 2-5 min. No requiere conversión hepática. La respuesta plaquetaria se recupera transcurridas 1-2 h. tras su infusión. Algunos ensayos clínicos en fase III en pacientes sometidos a intervención coronaria percutánea no han demostrado su superioridad en comparación con el clopidogrel (10). Sin embargo, a pesar de la falta de impacto clínico, la posibilidad de administrarlo vía parenteral y su reversibilidad lo hacen un candidato atractivo y fiable para pacientes de riesgo.

\section{Antagonistas del receptor de la glucoproteína IIb/IIIa}

Estos fármacos inhiben el receptor de la glucoproteína IIb/IIIa (GP Ilb/IIIa) y se unen a las cadenas de fibrinógeno en el último paso del proceso de agregación plaquetaria, ya que presentan un importante efecto antiagregante. Todos los fármacos de este grupo se administran por vía endovenosa y tienen una acción inmediata, por lo que no se utilizan en la prevención primaria. La inhibición de los receptores de la GP IIb/IIla se lleva a cabo a través de dos mecanismos: directo no competitivo con bloqueo permanente del receptor o por inhibición irreversible con competencia con el fibrinógeno impidiendo la agregación plaquetaria. Dentro de este grupo se encuentran: Eptifibatide, Tirofiban, Abciximab.

Actualmente se están desarrollando multiplicidad de moléculas inhibidoras de las GP IIb/IIla para administración por vía oral (xemilofiban, sibrafiban, orbofiban). La experiencia global demuestra unos resultados preliminares en principio perjudiciales, que requieren ulterior investigación y análisis (11).

\section{Inhibidores del receptor de la trombina}

En la actualidad existen dos fármacos inhibidores del receptor PAR-1 de la trombina, vorapaxar y atopaxar, ambos aún en fase de desarrollo clínico. Su administración es por vía oral. El vorapaxar se absorbe rápidamente y muestra mayor afinidad para PAR1 que el atopaxar. Alcanza la máxima acción antiplaquetaria a las $2 \mathrm{~h}$, mientras que el atopaxar es algo más tardío (3,5 h). El vorapaxar tiene una vida media larga, estimada en hasta $311 \mathrm{~h}$, mientras que la del atopaxar es de solo $23 \mathrm{~h}$ (12).

\section{USOS DE LOS ANTIAGREGANTES PLAQUETARIOS}

En medicina, dada la participación de las plaquetas, tanto en las etapas precoces de la formación de placas de ateroma (aterogénesis) como en la trombosis arterial, el uso de antiagregantes plaquetarios es muy relevante en la prevención primaria y secundaria de enfermedades cardiovasculares, como las trombosis 
arteriales especialmente el infarto agudo de miocardio (IAM), así como de trastornos cerebrovasculares.

La utilización de antiagregantes plaquetarios se ha demostrado como una importante medida de prevención primaria, es decir antes de que se haya producido un accidente trombótico, y aplicable a las personas con riesgo moderado o alto de sufrir un episodio coronario o accidente cerebrovascular. Las situaciones que constituyen en factor de riesgo en prevención primaria son: la insuficiencia renal crónica, la diabetes, la hipertensión, el tabaquismo y todo ello unido a la edad avanzada del paciente. Se aconseja el uso de ácido acetilsalicílico o, en caso de alergia o intolerancia, el clopidogrel.

La prevención secundaria es el conjunto de intervenciones que se dirigen a los pacientes que han sufrido un evento cardiovascular o accidente cerebrovascular, y tiene como objetivo evitar la repetición de un nuevo episodio, o en el caso de que éste suceda, que sea lo menos grave posible. Se aconseja en pacientes que padecen cardiopatía isquémica crónica (tipo angina de pecho o infarto de miocardio antiguo con o sin revascularización independientemente del tipo, percutánea o quirúrgica), pacientes con fibrilación auricular, miocardiopatía dilatada con disfunción ventricular severa y enfermedad arterial periférica.

Tanto en prevención primaria como secundaria, el antiagregante más utilizado es el AAS, en dosis preventivas de 75-150 mg/día; las dosis terapéuticas cuando se requiere un efecto antitrombótico inmediato son de $300 \mathrm{mg} /$ día. En los casos de alergia o intolerancia al AAS, el clopidogrel el fármaco antiagregante de elección.

\section{MECANISMO DE ACCIÓN Y CLASIFICACIÓN DE LOS ANTICOAGULANTES ORALES}

Durante décadas, la anticoagulación oral en el tratamiento y prevención de la tromboembolia venosa ha sido posible gracias a la utilización de fármacos antagonistas de la vitamina K (AVK). Los AVK son fármacos de manejo complejo. Su margen terapéutico es estrecho y requieren monitorización periódica para conseguir mantener unos niveles razonables de seguridad y eficacia, y presentan múltiples interaccio- nes con otros fármacos, con la ingesta de vitamina $\mathrm{K}$ o alcohol, con enfermedades intercurrentes y otros factores. En 1983, la OMS introdujo el índice INR (International Normalized Ratio) para estandarizar el nivel anticoagulante de estos fármacos antagonistas de la vitamina $\mathrm{K}$.

La investigación para encontrar una alternativa a los AVK se ha concentrado en el desarrollo de un anticoagulante oral con amplio margen terapéutico y escasa variabilidad intraindividual e interindividual que pueda administrarse con una dosis fija sin necesidad de monitorizar sistemáticamente la coagulación y con pocas interacciones.

En la actualidad, existen tres inhibidores directos del factor X activado (rivaroxabán, apixabán y edoxabán) y un inhibidor directo de la trombina (dabigatrán) con indicaciones aprobadas en profilaxis y tratamiento antitrombótico en diferentes situaciones (13). Aunque todavía no hay una experiencia amplia sobre su uso en la vida real, sobre todo a largo plazo, su seguridad y su eficacia han sido demostradas y están empezando a ser utilizados de forma habitual en sustitución de los AVK. Un problema general de estos de fármacos que no precisan control puede ser una menor adherencia de los pacientes al tratamiento (17).

Los anticoagulantes orales usados en la actualidad son:

- Heparina. Es un mucopolisacárido que se une a la antitrombina III, que actúa como un inhibidor potente de la trombina, y los factores IX, X, XI y XII de la coagulación. La administración se realiza por vía parenteral cada 24 horas. Debido a la desventaja de forma de administración, así como los efectos secundarios en periodos de tiempo superiores a 6 meses, como son la trombocitopenia y osteoporosis, su uso se reduce como terapia puente a los anticoagulantes orales en cirugía y para inmovilizaciones prolongadas (18).

- Acenocumarol (Sintrom ${ }^{\circledR}$ ) y Warfarina (Aldocumar®). En España, se utiliza principalmente el acenocumarol. Son fármacos antagonistas de la vitamina $\mathrm{K}$, que actúan reduciendo la síntesis hepática de los factores de coagulación II, VII, IX, X, así como la proteína C y S. Su efecto no es inmediato y requiere varios días para alcanzarlo, con una vida media plasmática entre 10-24 h. 
- Rivaroxabán (Xarelto $\left.{ }^{\circledR}\right)$, Apixaban (Eliquis®), Edoxaban (Lixiana $\left.{ }^{\circledR}\right)$. Los tres fármacos son inhibidores competitivos y reversibles del factor de coagulación Xa.

- Dabigatrán (Pradaxa $\left.{ }^{\circledR}\right)$. Se trata de un profármaco inhibidor reversible de la trombina, que actúa inhibiendo la transformación de fibrinógeno a fibrina, impidiendo la formación del trombo.

\section{USO DE LOS ANTICOAGULANTES ORALES}

El empleo de los anticoagulantes orales se centra en la prevención de la tromboembolia en pacientes con válvulas cardíacas protésicas, en la profilaxis de tromboembolia venosa en cirugía ortopédica programada (prótesis de rodilla o cadera), en el tratamiento de la trombosis venosa profunda (TVP) y de la embolia pulmonar (EP), y prevención de las recurrencias de la TVP y de la EP en pacientes adultos y en la prevención del ictus y la embolia sistémica en pacientes con fibrilación auricular $(19,20)$.

\section{PROTOCOLOS DE ACTUACIÓN ANTE PACIENTES ANTIAGREGADOS Y ANTICOAGULADOS}

Para valorar la continuidad o suspensión del tratamiento antitrombótico de un paciente ante la realización de un procedimiento dental invasivo, debemos de tener en cuenta el riesgo hemorrágico del tratamiento dental y, por otro lado, el riesgo trombótico del paciente.

La extracción dental simple o quirúrgica, la colocación de implantes sin injerto óseo y colgajos muy extensos, así como procedimientos de cirugía bucal de corta duración $\leq 45$, son considerados procedimientos de cirugía menor con riesgo bajo o normal de sangrado (21-23). Por el contrario, procedimientos de cirugía bucal mayor, la realización de extracciones dentales múltiples, intervenciones quirúrgicas bucales que superan los 45 minutos o cirugía del cáncer de cabeza y cuello se consideran como tratamientos del alto riesgo de sangrado (24).

La determinación del riesgo trombótico debe ser evaluada por el médico especialista del paciente, ya que son muchos los factores que lo condicionan, por lo que la decisión de continuar, retirar o sustituir el tratamiento antitrombótico la determinará el médico especialista, y no el odontoestomatólogo o el paciente (25). Los pacientes con riesgo trombótico bajo son aquellos con tratamiento antiagregante como mecanismo de prevención primaria de accidente cardiovascular.

En el manejo del paciente que se le va a realizar una extracción dental, independientemente del tipo de tratamiento antitrombótico, debemos de llevar a cabo una serie de consideraciones y medidas locales encaminadas a reducir el riesgo hemorrágico, las cuales serían las siguientes:

- Periodo preoperatorio:

- Es necesario reducir los irritantes y la inflamación de los tejidos blandos. Los pacientes deben de mantener una buena higiene oral en la semana previa a la extracción y si es necesario habrá que realizar procedimientos de tartrectomía. Son recomendables la prescripción de enjuagues de clorhexidina al 0,2\% dos veces al día durante una semana.

\section{- Periodo operatorio:}

- Es recomendable realizar las extracciones por la mañana y al principio de la semana, para que el paciente tenga la posibilidad de acudir a su odontólogo ante la aparición de complicaciones hemorrágicas.

- La técnica quirúrgica debe ser lo más correcta y atraumática posible. Si fuera necesario realizar algún colgajo, éste debe ser mucoperióstico.

- Tras la exodoncia se debe realizar un curetaje meticuloso del alveolo para eliminar todo el tejido inflamatorio y de granulación para reducir el sangrado de la herida.

- Si se considera necesario, se pueden colocar apósitos en el interior del alveolo de tipo esponjas de colágeno, fibrina o celulosa oxidada.

- Se recomienda la sutura del alveolo, para conseguir un cierre primario de la herida.

- Por último, se recomienda la compresión con una gasa seca durante una hora, o impregnada en sustancias antifibrinolíticas como el ácido tranexámico.

- Mantener al paciente 45-60 minutos en la consulta para controlar el sangrado antes de que se vaya. 


\section{- Periodo postoperatorio:}

- Evitar la realización de enjuagues durante las primeras 24 horas.

- Reposo, evitando la realización de ejercicio físico.

- Evitar comidas o bebidas calientes.

- En caso de sangrado, se recomienda colocar una gasa impregnada en antifibrinolíticos, como el ácido tranexámico. Algunos autores son partidarios de realizar enjuagues con el ácido tranexámico durante 60 segundos 2-3 veces al día (26), aunque otros autores no comparten esta opinión (27), y señalan que el enjuague aumenta el riesgo de disolución del coágulo y sangrado.

- Si el sangrado no cesa pasados 20-30 minutos el paciente debe ponerse en contacto con el odontólogo para llevar a cabo las medidas necesarias que detengan la hemorragia.

En el caso de los pacientes en tratamiento con antiagregantes plaquetarios, si el paciente presenta un riesgo trombótico bajo, es decir, su tratamiento antiagregante está indicado por prevención primaria, es posible la suspensión del tratamiento antitrombótico unos días previos a la intervención sin aumento del riesgo trombótico, y reanudarlo al día siguiente, 7 días en el caso de la aspirina y 5 días para el clopidogrel. En los casos de que el riesgo trombótico sea moderado o alto, se recomienda continuar con el tratamiento antiagregante y aplicar medidas locales de control de hemorragia si fuera necesario.

Son varios los trabajos que han puesto de manifiesto como el mantenimiento de la terapia antiagregante no condiciona un aumento de complicaciones hemorrágicas tras la realización de extracciones dentales, y en el caso de aparecer alguna de éstas, la aplicación de medidas locales como la utilización de apósitos antifibrinolíticos en el interior del alveolo, la sutura del mismo, y la compresión con gasa seca o impregnada en ácido tranexámico, son suficientes para el control de las mismas.

Trabajos recientes han demostrado que el riesgo de sangrado inducido por los fármacos antiagregantes ha sido exagerado, mientras que el riesgo trombótico por suspensión del tratamiento ha sido subestimado $(28)$. Distintos estudios $(29,30)$ señalan que la realización de extracciones dentales y procedimientos de cirugía bucal menor sin suspensión del tratamiento antiagregante no condicionaban un aumento del sangrado respecto a pacientes sin tratamiento antiagregante, y que la aplicación de medidas locales hemostáticas eran suficientes para controlar la hemorragia postquirúrgica.

Cardona y cols. (29) refieren que existe una relación estadísticamente significativa entre el número de dientes extraídos y la subsecuente hemorragia, y recomiendan no extraer más de tres dientes en el mismo acto, los cuáles deben ser adyacentes. Enfatizan que el empleo de medidas locales son apropiadas para el control de la hemorragia postextracción.

Al-Murabak y cols. (31) no consideran que la sutura del alveolo sea un procedimiento necesario en estos pacientes, sólo en los casos de extracciones múltiples o quirúrgicas. Por el contrario, otros autores sí son partidarios de la sutura del alveolo en la extracción simple como medida local protocolizada en el control de la hemorragia $(32,33)$.

A pesar de que se considera seguro la suspensión del tratamiento antiagregante en pacientes de bajo riesgo trombótico, los protocolos de actuación aconsejan la no suspensión del mismo, ya que el empleo de medidas hemostáticas locales van a ser suficientes ante una posible complicación hemorrágica.

Los protocolos de actuación en pacientes anticoagulados que van a ser sometidos a procedimientos de cirugía bucal han ido evolucionando con el tiempo. Al principio éstos recomendaban la sustitución del anticoagulante oral por una heparina de bajo peso molecular. En la actualidad, las extracciones dentales simples y quirúrgicas, se consideran procedimientos de cirugía menor con bajo riesgo de sangrado, por lo que son numerosos los estudios que recomiendan no suprimir el tratamiento anticoagulante (34-36).

La mayoría de los pacientes anticoagulados se encuentran en tratamiento con antagonistas de la vitamina K. El manejo de estos fármacos es complejo, las múltiples intereacciones con otras sustancias hacen necesaria una monitorización del anticoagulante previo al procedimiento dental, por lo que debemos solicitar al paciente que se le realice una de- 
terminación del INR actual, lo ideal sería realizarla en el mismo día de la exodoncia, pero se admiten valores de INR que no superen las 72 horas de antigüedad. El rango de INR apropiado para realizar el procedimiento dental oscila de 2 a 4 , aunque es ampliamente aceptado que el valor óptimo es 2,5, porque este valor minimiza el riesgo de sangrado y trombosis (34).

En la actualidad, la comunidad científica en base a los resultados obtenidos en estudios clínicos recomiendan que para extracciones dentales o cirugía mínimamente invasiva no se retire la anticoagulación oral, mientras se mantenga un INR entre 2 y 4; en intervenciones en las que se espere un riesgo de sangrado significativo el INR debe ser $<3$ y finalmente diferir la intervención y consulta con el médico especialista para ajuste de la dosis anticoagulante en los casos de INR $>4(30,31,37-41)$.

Potoski y cols. (34) señalan en diferentes estudios revisados para procedimientos menores como extracciones dentales, que la aparición de trombosis oscila entre el $0,02 \%$ y $1 \%$, mientras que el riesgo de sangrado entre el $0 \%$ y 3,5\%. La gravedad de las complicaciones son más importantes en la aparición de la trombosis, que en el caso de hemorragia. En este sentido, Wahl (42) analizó 2014 procedimientos de cirugía bucal en pacientes con tratamiento anticoagulante, de 493 pacientes que habían suspendido el tratamiento anticoagulante, 5 pacientes desarrollaron complicaciones tromboembólicas, que conllevaron el fallecimiento de cuatro pacientes. Por ello, la mayoría de los estudios recomiendan en la actualidad, que en el caso de la extracción dental de uno o dos dientes, no se debe retirar el anticoagulante oral $(43,44)$. Un estudio metaanalítico realizado por Rodríguez-Cabrera y cols. (44) sobre 1.194 pacientes anticoagulados sometidos a una o varias extracciones dentales puso de manifiesto como sólo en un $6,95 \%$ de los casos se produjo un sangrado postoperatorio, que fue controlado en la mayoría de las situaciones mediante medidas locales.

Ante la realización de extracciones dentales múltiples, donde el riesgo de sangrado aumenta, Spyropoulos y cols. (24) recomiendan no extraer más de tres dientes en el mismo acto y, a ser posible, adyacentes.
Por otro lado, en aquellas situaciones donde el tipo procedimiento quirúrgico bucal conlleva un mayor sangrado, con niveles de INR $>3,5$, los protocolos de actuación aconsejan las suspensión del anticoagulante 2-3 días previos a la cirugía y su reemplazo por una heparina de bajo peso molecular y reinicio de la dosis anticoagulante a las 12 horas de la intervención (45).

Los protocolos de actuación en los pacientes en tratamiento con los nuevos anticoagulantes orales son muy escasos, debido a su reciente introducción. Algunos trabajos $(22,46,47)$ indican que no existe necesidad de suspender o modificar la anticoagulación en el caso de intervenciones dentales de bajo o normal riesgo hemorrágico. En el caso de alto riesgo hemorrágico, la recomendación sería suspender el anticoagulante 24 horas antes de la intervención y reanudarlo 24 horas después. En cualquier situación, se considera necesario el empleo de medidas locales para el control del sangrado.

\section{BIBLIOGRAFÍA}

1. Vilahur G, Badimon J, Bugiardini R, Badimon L. Perspectives: The burden of cardiovascular risk factors and coronary heart disease in Europe and worldwide. Eur Heart J 2014;16:A7-A11.

2. Navarro JL, César JM, Fernández MA, Fontcuberta J, Reverter JC, Gol-Freixa J. Morbilidad y mortalidad en pacientes con tratamiento anticoagulante oral. Rev Esp Cardiol 2007;60: 1226-32.

3. Weiss HJ, Aledort LM. Impaired plateletconnective-tissue reaction in man after aspirin ingestion. Lancet 1967; 2:495-7.

4. Catella-Lawson F, Reilly MP, Kapoor SC, Cucchiara AJ, DeMarco S, Tournier B, et al. Cyclooxygenase inhibitors and the antiplatelet effects of aspirin. $\mathrm{N}$ Engl J Med 2001;345:1809-17.

5. Tavares J, de Camargo Carvalho A. Antiplatelet Agents in Acute Coronary Syndrome. Int J Atherosclerosis 2007;2(4):251-64. 
6. Ruiz Mori E. Antiagregantes plaquetarios. Rev Per Cardiol 2006;32(1):26-38.

7. Yusuf S, Zhao F, Mehta SR, Chrolavicius S, Tognoni G, Fox KK. Effects of clopidogrel in addition to aspirin in patients with acute coronary syndromes without st-segment elevation. $\mathrm{N}$ Engl J Med 2001;345:494-502.

8. Storey RF, Angiolillo DJ, Patil SB, Desai B, Ecob $\mathrm{R}$, Husted $\mathrm{S}$, et al. Inhibitory effects of ticagrelor compared with clopidogrel on platelet function in patients with acute coronary syndromes: The PLATO (Platelet Inhibition And Patient Outcomes) platelet substudy. J Am Coll Cardiol 2010;56: 1456-62.

9. Teng R. Pharmacokinetic, pharmacodynamic and pharmacogenetic profile of the oral antiplatelet agent ticagrelor. Clin Pharmacokinet 2012;51: 305-18.

10. Bhatt DL, Lincoff AM, Gibson CM, Stone GW, McNulty S, Montalescot G, et al. Intravenous platelet blockade with cangrelor during PCI. $\mathrm{N}$ Engl J Med 2009;361:2330-41.

11. Sitges M, Bosch X, Sanz G. Eficacia de los bloqueadores de los reseptores plaquetarios IIb/IIla en los síndromes coronaries agudos. Rev Esp Cardiol 2000;53:422-39.

12. Badimon L, Medieta G, Vilahur G. Diferencias en los mecanismos de acción de los nuevos antiagregantes: ¿cómo actúan? Rev Esp Cadiol Supl 2014;14(A):3-9.

13. Wittkowsky AK. Novel oral anticoagulants and their role in clinical practice. Pharmacotherapy 2011;31:1175-91.

14. Connolly SJ, Ezekowitz MD, Yusuf S, Eikelboom $\mathrm{J}$, Oldgren J, Parekh A, et al. for the RE-LY Steering Committee and Investigators. Dabigatran versus warfarin in patients with atrial fibrillation. N Engl J Med 2009;361:1139-51.

15. Patel MR, Mahaffey KW, Garg J, Pan G, Singer DE, Hacke W, et al. for the ROCKET AF Investigators.
Rivaroxaban versus warfarin in nonvalvular atrial fibrillation. N Engl J Med 2011;365:883-91.

16. Connolly SJ, Eikelboom J, Joyner C, Diener HC, Hart R, Golitsyn S, et al, for the AVERROES Steering Committee and Investigators. Apixaban in patients with atrial fibrillation. $\mathrm{N}$ Engl $\mathrm{J}$ Med 2011;364:806-17.

17. Pengo V, Crippa L, Falanga A, Finazzi G, Marongiu F, Palareti G, et al. Questions and answers on the use of dabigatran and perspectives on the use of other new oral anticoagulants in patients with atrial fibrillation. A consensus document of the Italian Federation of Thrombosis Centers (FCSA). Thromb Haemost 2011;106:868-76.

18. Hong CH, Napeñas JJ, Brennan MT, Furney SL, Lockhart PB. Frequency of bleeding following invasive dental procedures in pa- tients on lowmolecular-weight heparin ther-apy. J Oral Maxillofac Surg 2010;68:975-9.

19. Mateo J. Nuevos anticoagulantes orales y su papel en la práctica clínica. Rev Esp Cardiol Supl 2013;13(C):33-41.

20. Sorensen R, Gislason G. Tratamiento antitrombótico combinado triple: arriesgado pero a veces necesario. Rev Esp Cardiol 2014;67:171-5.

21. Sánchez-Martínez M, Manzano-Fernández S, Valdés M, Marín F. ¿Por qué debemos estratificar el riesgo hemorrágico junto con el isquémico en el síndrome coronario agudo? Rev Esp Cadiol Supl 2014;14(A):10-7.

22. Mingarro de León A, Chaveli-López B, GavaldáEsteve C. Dental management of patiens receiving anticoagulant and/or antiplatelel treatment. J Clin Exp Dent 2014;6(2):e155-61.

23. Madrid C, Sanz M. What influence do anticoagulants have on oral implant therapy? A systematic review. Clin Oral Implants Res 2009. Suppl 4:96-106.

24. Spyropoulos AC, Douketis JD. How I treat anticoagulated patients undergoing an elective 
procedure or surgery. Blood 2012;120:295462.

25. Martínez López F, Oñate Sánchez R, Arrieta Blanco J, Oñate cabrerizo D, Cabrerizo Merino M. Clinical diseases with thrombotic risk and their pharmacologycal treatment: How they change the therapeutic attitude in dental treatments. Med Oral Patol Oral Cir Bucal 2013 Nov;18(6):e888-e95.

26. Carter G, Goss A. Tranexamic acid mouthwash a prospective randomized study of a 2 -day regimen vs 5 -day regimen to prevent postoperative bleeding in anticoagulated patients requiring dental extractions. Int J Oral Maxillofac Surg 2003;32: 504-7.

27. Ferrieri GB, Castiglioni S, Carmagnola D, Cargnel M, Strohmenger L, Abati S. Oral surgery in patients on anticoagulant treatment without therapy interruption. J Oral Maxillofac Surg 2007; 65:1149-54.

28. Aframian DJ, Lalla RV, Peterson DE. Management of dental pa- tients taking common hemostasisaltering. Oral Surg Oral Med Oral Pathol Oral Radiol Endod 2007;103:S45.e1-11.

29. Cardona-Tortajada F, Sainz-Gómez E, FigueridoGarmencia J, Litón de Robles-Adsuar A, MorteCasabó A, Giner-Muñoz F, Artázcoz-Osés J, Vidán-Lizari J.Dental extractions in patients on antiplatelet therapy. A study conducted by the Oral Health Department of the Navarre Health Service (Spain). Med Oral Patol Oral Cir Bucal 2009 Nov 1;14(11):e588-92.

30. Madan GA, Madan SG, Madan G, Madan AD. Minor oral surgery without stopping daily lowdose aspirin therapy: a study of 51 patients. J Oral Maxillofac Surg 2005;63:1262-5.

31. Al-Mubarak S, Rass MA, Alsuwyed A, Alabdulaaly A, Ciancio S. Thromboembolic risk and bleeding in patients maintaining or stopping oral anticoagulant therapy during dental extraction. $\mathrm{J}$ Thromb Haemost 2006;4:689-91.

32. Morimoto Y, Niwa H, Minematsu K. Hemostatic management of tooth extractions in patients on oral antithrombotic therapy. J Oral Maxillofac Surg 2008;66:51-7.

33. Madan GA, Madan SG, Madan G, Madan AD. Minor oral surgery without stopping daily lowdose aspirin therapy: a study of 51 patients. J Oral Maxillofac Surg 2005;63:1262-5.

34. Pototski M, Amenábar JM. Dental management of patients receiving anticoagulation or antiplatelet treatment. J Oral Sci 2007;49:253-8.

35. Jiménez Y, Poveda R, Gavaldá C, Margaix $M$, Sarrión G. An update on the management of anticoagulated patients programmed for dental extractions and surgery. Med Oral Patol Oral Cir Bucal 2008;13:e176-9.

36. Cañigral A, Silvestre FJ, Cañigral G, Alós M, Garcia-Herraiz A, Plaza A. Evaluation of bleeding risk and measurement methods in dental patients. Med Oral Patol Oral Cir Bucal 2010;15: e863-8.

37. Evans IL, Sayers MS, Gibbons AJ, Price G, Snooks H, Sugar AW. Can warfarin be continued during dental extraction? Results of a randomized controlled trial. $\mathrm{Br} \mathrm{J}$ Oral and Maxillofac Surg 2002;40:248-52.

38. Méndez C, Cisneros L. Control del sangrado postoperatorio en pacientes anticoagulados empleando colutorios de ácido tranexámico. Odontología Sanmarquina 2005;8:14-9.

39. Sacco R, Sacco M, Carpenedo M, Mannucci PM. Oral surgery in patients on oral anticoagulant therapy: a randomized comparison of different intensity targets. Oral Surg Oral Med Oral Pathol Oral Radiol Endod 2007;104:e18-e21.

40. Bajkin BV, Popovic SL, Selakovic SD. Randomized, Prospective Trial Comparing Bridging Therapy Using Low-Molecular-Weight Heparin With Maintenance of Oral Anticoagulation During Extraction of Teeth. J Oral Maxillofac Surg 2009; 67:990-5.

41. Lozano I, Martín D, Torres F, Avanzas P, Rondán J, García-Ruiz JM, Hernández E, Bayón J, Vegas 
JM, Espolita A. Grado de conocimiento de stents coronarios, trombosis y doble antiagregación entre los odontólogos en España. Rev Esp Cardiol 2009;62(2):153-7.

42. Wahl MJ. Dental surgery in anticoagulated patients. Arch Intern Med 1998;158:1610-6.

43. Rodríguez-Cabrera MA, Barona-Dorado C, LecoBerrocal I, Gómez-Moreno G, Martínez-González JM. Extraction without eliminating anticoagulant treatment: a literatura review. Med Oral Patol Oral Cir Bucal 2011;16:e800-4.

44. Jiménez Y, Poveda R, Gavaldá C, Margaix M, Sarrión G. An up- date on the management of anticoagulated patients programmed for dental extractions and surgery. Med Oral Patol Oral Cir Bucal 2008;13:E176-9.
45. Firriolo FJ, Hupp WS. Beyond warfarin: the new generation of oral anticoagulants and their implications for the management of dental patients. Oral Surg Oral Med Oral Pathol Oral Radiol 2012;113:431-41.

46. Little JW. New oral anticoagulants: will they replace warfarin? Oral Surg Oral Med Oral Pathol Oral Radiol 2012;113:575-80

\section{CORRESPONDENCIA}

Prof. José María Martínez-González

Departamento de Medicina y Cirugía Bucofacial Facultad de Odontología

Universidad Complutense de Madrid

Correo electrónico: jmargo@ucm.es 